\title{
不同光照和水分条件下鬼针草属入侵种与本地种 生长、光合特征及表型可塑性的比较
}

\author{
潘玉梅 唐赛春 ${ }^{*}$ 韦春强 李象钦 \\ (广西壮族自治区中国科学院广西植物研究所广西植物功能物质研究与利用重点实验室, 广西桂林 541006)
}

\begin{abstract}
摘要: 为探讨鬼针草属(Bidens)入侵种的入侵性, 利用同质园种植实验比较了该属入侵种三叶鬼针草(Bidens pilosa)和大狼耙草(B. frondosa) 与本地种金盛银盘(B. biternata)和狼耙草(B. tripartita)在光照与水分交互作用下的形 态、生长、生物量分配、光合特征及其表型可塑性的差异。结果表明: 入侵种的株高和生物量在低光低水条件下 与本地种相似, 在有利的光照和水分条件(高光高水)下显著高于本地种, 相对生长速率在高光条件下均高于本地 种。入侵种在高光处理下增加了对地下部分的资源投入, 在低光处理下增加了对叶的投入, 且低光低水条件下比 叶面积显著高于本地种。这些特性可能提高了入侵种对资源的捕获和利用能力, 使其既能耐受不利的环境, 又能 在有利的条件下表现最大化。入侵种和本地种的形态、生长和光合生理等参数对水分变化的可塑性指数均较小, 对 光照变化的可塑性指数均较大。入侵种的多数变量对光照响应的可塑性指数大于本地种, 较大的表型可塑性可能 促进其成功入侵。另外, 入侵种和本地种的光合生理参数无显著差异。相对于光合特征, 形态、生长、生物量分 配和表型可塑性等可能对鬼针草属入侵种的入侵性更为重要。
\end{abstract}

关键词: 鬼针草属; 入侵种; 本地种; 生长特征; 光合特征; 表型可塑性

\section{Comparison of growth, photosynthesis and phenotypic plasticity between invasive and native Bidens species under different light and water condi- tions}

\author{
Yumei Pan, Saichun Tang*, Chunqiang Wei, Xiangqin Li \\ Guangxi Key Laboratory of Functional Phytochemicals Research and Utilization, Guangxi Institute of Botany, Guangxi \\ Zhuang Autonomous Region and Chinese Academy of Sciences, Guilin, Guangxi 541006
}

\begin{abstract}
To explore the traits related to the invasiveness of exotic Bidens species in China, we compared the morphology, growth, biomass allocation, photosynthesis, and phenotypic plasticity of two invasive Bidens species (B. pilosa and B. frondosa) with two native congeners (B. biternata and B. tripartita) under different light and water conditions in a common garden. The results showed that the invasive and native species displayed similar plant heights and total biomass under unfavorable conditions (i.e. low light and low water treatment). However, under favorable light and water conditions (i.e. high light and high water treatment), the invasive species showed significantly greater plant height and total biomass than the native congeners. Furthermore, in high light treatments, the relative growth rate of the invasive species was higher than that of the native species. The invasive species allocated more resources to root biomass at high light levels compared to low light levels, while they allocated more resources to leaf biomass at low light levels compared to high light levels. Specific leaf area of the invasive species was greater than that of the native congeners under low light conditions. These traits may enhance the abilities of invasive species to capture and utilize resources, enabling them to withstand adverse environmental conditions or to respond more positively to favorable conditions. The phenotypic plasticity indices of invasive and native species for morphology, growth, and photosynthetic parameters were low for water availability and high for light intensity. However, for most variables, invasive species showed a higher phenotypic plasticity index than native congeners, and this may contribute
\end{abstract}

收稿日期: 2016-12-26; 接受日期: 2017-07-01

基金项目: 国家自然科学基金(31460165, 31260154)

* 通讯作者 Author for correspondence. E-mail: tangs@gxib.cn 
to their invasion success. In addition, there were no significant differences for photosynthetic parameters between invasive and native Bidens species under any treatment. In conclusion, this study shows that morphology, growth, biomass allocation, and phenotypic plasticity may play more important roles than photosynthetic parameters in the success of invasive Bidens species.

Key words: Bidens; invasive species; native species; growth traits; photosynthetic characteristics; phenotypic plasticity

作为全球变化的重要组成部分, 生物入侵已成 为人类面临的巨大挑战。外来物种的入侵性和生境 的可入侵性是入侵生物学的两大基本问题。外来物 种的入侵性常表现为比本地种或外来非入侵种具 有更高的相对生长速率 (Grotkopp \& Rejmánek, 2007; Zheng et al, 2009)、更大的比叶面积(Deng et al, 2004; 宋莉英等, 2009)和光合速率(McDowell, 2002; 王晓红和纪明山, 2013), 这些都是与资源捕获和利 用效率相关的形态、生长和生理性状。并且, 入侵 植物通常具有较高的表型可塑性(Daehler, 2003; Mozdzer \& Megonigal, 2012), 可使其在各种环境条 件下保持较高的适合度(耿宇鹏, 2004)。比较入侵种 和近缘本地种的性状差异对揭示入侵种成功入侵 的机制具有重要作用。

三叶鬼针草(Bidens pilosa)和大狼耙草(B. frondos) 是菊科鬼针草属的入侵种, 这两个入侵种具异 型种子, 且种子有芒刺、千粒重较大、萌发率可达 $90 \%$ 以上(洪岗等, 2004; 周超群等, 2015), 与我国 入侵严重的同科入侵植物紫茎泽兰(Eupatorium adenophorum) (赵相健等, 2009)、飞机草(Chromolaena odoratum) (全国明等, 2011; 韦春强等, 2012)、加拿 大一枝黄花(Solidago canadensis) (黄华和郭水良, 2005)等的种子特性有显著差异。目前, 对鬼针草属 入侵植物的研究主要集中在化感作用(郭艾英等, 2010; 毛丹鹃等, 2010; 间小红等, 2012)、繁育系统 (郝建华等, 2009; Yan et al, 2016)以及生长和竞争对 养分的响应方面(潘玉梅等, 2012a, b; Pan et al, 2016)。这两种入侵植物与同属本地种金盏银盘(B. biternata)和狼耙草(B. tripartita)有着相似的生活史 和分布生境, 与同属本地种相比, 它们是否具有更 优越的形态、生长和生理性状, 较少有资料报道。

光照和水分直接影响植物的生理过程和生长, 是影响植物分布的重要因子(Quero et al, 2006)。 Zheng等(2009)研究表明, 入侵植物紫茎泽兰在高 光下具有较大的生物量, 而在低光下具有较大的比
叶面积; Geng等(2006)研究表明, 在水分充足时入 侵植物喜旱莲子草(Alternanthera philoxeroides)具 有较大的生物量和比叶面积, 而在水分不足时具有 较高的根生物量比。然而, 生境中的生态因子往往 具有综合作用, 多因子的交互作用对植物常产生不同 的影响 (Albert et al, 2011)。例如, 柔枝莠竹 (Microstegium vimineum) 的生物量在高光高水时显 著增加, 低光低水时显著减少; 而比叶面积在低光 高水时显著增加, 在高光低水时显著减少(Droste et al，2010)。了解光照和水分交互变化时入侵植物是 否保持较高的适合度, 其形态、生长和生理等性状 如何响应光照和水分的同时变化, 对于揭示入侵植 物的入侵性具有重要意义。

为此, 本研究通过同质园种植实验, 设置光照 和水分交互的条件, 比较鬼针草属两个入侵种(三 叶鬼针草和大狼耙草)与同属本地种(金戞银盘和狼 耙草)的形态、生长、光合特性及其表型可塑性的差 异, 旨在探讨: (1)与同属本地种相比, 鬼针草属入 侵种是否具有优越的形态、生长和生理性状; (2)光 照和水分交互变化时, 入侵植物是否保持较高的适 合度, 其形态、生长和生物量分配、生理等性状如 何响应光照和水分的同时变化; (3)与同属本地种相 比, 鬼针草属入侵种是否具有较高的表型可塑性。

\section{1 材料与方法}

\section{1 实验材料}

本实验于2013年在广西壮族自治区中国科学 院广西植物研究所的温室大棚进行(位于桂林市雁 山区雁山镇, $25^{\circ} 04^{\prime} 49.6^{\prime \prime} \mathrm{N}, 110^{\circ} 18^{\prime} 01.8^{\prime \prime} \mathrm{E}, 170 \mathrm{~m}$ a.s.1.)。该地属中亚热带季风气候, 年平均气温 $17.8^{\circ} \mathrm{C}$, 最冷月 1 月平均温度 $5.8^{\circ} \mathrm{C}$, 最热月 7 月平均 温度 $28^{\circ} \mathrm{C}$; 年平均降水量 $1,949.5 \mathrm{~mm}$ 。

2012年10-11月，在桂林市附近收集入侵种三 叶鬼针草、大狼耙草及同属本地种金盏银盘和狼耙 草的种子, 每种植物的种子约来自于 20 株植株, 且 
植株相互间的距离不少于 $20 \mathrm{~m}$ 。所有材料的种子分 别装在纸袋里保存备用。

\section{2 方法}

2013年5月, 在大棚内将每种植物的种子分别 种在装有相同土质的塑料盆内进行育苗。待每种植 物幼苗长至约 $10 \mathrm{~cm}$ 高时, 选取具有相似株高和基 茎的植株移植到装有相同表层土的塑料盆内(内径 $24 \mathrm{~cm}$, 底径 $16 \mathrm{~cm}$, 高 $18 \mathrm{~cm})$, 每盆 1 株。土壤 $\mathrm{pH}$ 值 为 6.51 , 主要成分为: 有机碳 $18.80 \mathrm{~g} / \mathrm{kg}$, 总氮 $0.94 \mathrm{~g} / \mathrm{kg}$, 总磷 $0.84 \mathrm{~g} / \mathrm{kg}$, 总钾 $12.32 \mathrm{~g} / \mathrm{kg}$, 有效氮 $98 \mathrm{mg} / \mathrm{kg}$, 有效磷 $12 \mathrm{mg} / \mathrm{kg}$, 有效钾 $140 \mathrm{mg} / \mathrm{kg}$ 。所有 移植的幼苗在 $40 \%$ 的光照下适应生长 2 周后再进行 处理, 处理前每种植物幼苗分别选取10株烘干称重, 此10株总生物量的平均值作为计算相对生长速率 时该种植物的初始平均生物量。

设置 2 个光照水平和 2 个水分梯度的交互处理, 即高光高水、低光高水、高光低水和低光低水共 4 个处理, 每种植物每个处理设置 6 个重复。实验共种 植 96 盆 $($ 光 $\times$ 水 $\times$ 物种 $\times$ 重复 $=2 \times 2 \times 4 \times 6$ )。 光照处理参照Zou等(2009)的设计方法: 高光处理 即接受自然全光照; 低光处理是指覆盖两层黑色遮 阴网(约遮光 $80 \%$ )。水分处理参照Burns (2004)和王 坤等(2010)的设计方法: 高水处理是每天浇水至盆 内土壤水分饱和, 保持土壤湿润; 低水处理是待盆 内植株发生萎蒸时, 向盆内浇水至饱和。实验期间 对每个处理的植株每隔一周进行旋转和重新排列, 并及时灭虫除草。

在植株处于营养生长旺盛期(少数植株开始出 现花蕾)时, 用Li-6400光合仪(Li-COR, Lincoln, Nebraska, 美国)测量植物的光合-光响应曲线, 每个 处理每种植物测量 3 株。光合响应设置 13 个光辐射 梯度, 分别为: $2,000,1,500,1,200,1,000,800,600$, $400,200,150,100,50,20,0 \mu \mathrm{mol} \cdot \mathrm{m}^{-2} \cdot \mathrm{s}^{-1}$, 测量时间 为天气晴朗的上午 $8: 00-11: 30$, 根据 Cannell和 Thornley (1998)与Endo等(2001)的非直角双曲线模 型模拟每种植物的光合-光响应曲线, 并根据以下 光合响应曲线方程计算光合参数, 如最大净光合速 率 $\left(A_{\max }\right)$ 、光饱和点 $(L S P)$ 和光补偿点 $(L C P)$ 等。

$A=\frac{P_{\mathrm{si}} Q+A \max -\sqrt{\left(P_{\mathrm{si}} Q+A \max \right)^{2}-4 k P_{\mathrm{si}} Q A \max }}{2 k}-R_{\text {day }}(1)$
其中, $A$ 为净光合速率 $\left(\mu \mathrm{mol} \cdot \mathrm{m}^{-2} \cdot \mathrm{s}^{-1}\right) ; P_{\mathrm{si}}$ 为表观量子效 率 $\left(\mu \mathrm{mol} \cdot \mathrm{m}^{-2} \cdot \mathrm{s}^{-1}\right) ; A_{\max }$ 为最大净光合速率 $\left(\mu \mathrm{mol} \cdot \mathrm{m}^{-2} \cdot \mathrm{s}^{-1}\right) ; Q$ 为光合有效辐射 $\left(\mu \mathrm{mol} \cdot \mathrm{m}^{-2} \cdot \mathrm{s}^{-1}\right) ; k$ 为 光响应曲线曲角; $R_{\mathrm{day}}$ 为暗呼吸速率 $\left(\mu \mathrm{mol} \cdot \mathrm{m}^{-2} \cdot \mathrm{s}^{-1}\right)$ 。

每种植物每个处理随机选择3株进行叶面积的 测定(Li-3000叶面积测量仪, Li-COR, Lincoln, Nebraska，美国)，每个植株最多收集20片叶子，分别 烘干、称重, 计算比叶面积(叶面积/叶重)。这些叶 片的生物量最终计入到植株的总生物量进行分析。

实验从处理到最终收获共 $80 \mathrm{~d}$, 收获时记录不 同处理下每株植物的株高、分枝数, 并将植株的根、 茎和叶分离, 分别烘干称重, 计算总生物量。根据 Poorter (1999)的方法, 计算根生物量比(root mass ratio, $R M R$ )、茎生物量比(stem mass ratio, SMR)、叶 生物量比(leaf mass ratio, LMR) 和相对生长速率 (relative growth rate, $R G R$, 单位: $\mathrm{mg} \cdot \mathrm{g}^{-1} \cdot \mathrm{d}^{-1}$ )。

$$
R G R=\frac{\ln W_{2}-\ln \bar{W}_{1}}{\Delta t}
$$

其中, $W_{2}$ 为最终生物量; $\bar{W}_{1}$ 为平均初始生物量; $\Delta t$ 为生长期, 本研究以 $80 \mathrm{~d}$ 计算。并根据Valladares等 (2000)计算所测量参数的可塑性指数, 即某一变量 在不同的光照或水分条件下最大平均值和最小平 均值的差值与最大平均值的比。

\section{3 数据分析}

根据来源将 4 种植物分为入侵 (三叶鬼针草和大 狼耙草)和本地 (金戞银盘和狼耙草)两类, 物种作为 随机因子嵌套于物种来源中, 将光照、水分、物种 来源(即入侵和本地)作为固定因子, 对所测变量进 行多因素方差分析。利用独立样本 $t$-test分析不同处 理下所测变量在入侵种与本地种之间的差异性 $(P<$ 0.05), 利用One-way ANOVA分别分析入侵种和本 地种各变量在不同光照和水分处理下的差异性。所 有分析均在SPSS 13.0软件中进行, 生物量数据进 行开平方根转换以满足方差齐性。

\section{2 结果}

\section{1 光照和水分变化对入侵种与本地种形态、生长 参数的影响}

光照和水分对株高、生物量、比叶面积和相对 生长速率均有显著影响(表1)。入侵种和本地种的株 高、生物量及相对生长速率均随着光照和水分的减 少而降低; 比叶面积均随着光照的降低显著增加 
(图1)。光照与物种来源对形态和生长参数的交互影 响显著(表1), 说明入侵种与本地种的形态、生长参 数对光照变化的响应有差异, 而水分与来源对形态 和生长参数的交互影响不显著(表1), 说明它们对水 分变化的响应一致。入侵种的株高和生物量在高光 高水处理下显著高于本地种(图1a，c); 比叶面积在 低光低水处理下显著大于本地种(图1b); 除低光高 水处理外, 入侵种的相对生长速率在其他 3 种条件 下都显著高于本地种(图1d)。

\section{2 光照和水分变化对入侵种与本地种生物量分 配的影响}

光照对根生物量比和叶生物量比均影响显著, 水分对茎生物量比和叶生物量比影响显著(表1), 说 明在不同光照或水分处理间生物量分配存在差异; 光照与来源的交互作用对根生物量比、茎生物量比 和叶生物量比有显著影响(表1), 说明入侵种与本地 种各构件生物量分配对光照变化的响应不同。入侵 种的根生物量比随着光照的降低显著降低, 对水分 变化的响应不显著(图1e); 茎生物量比在高光高水 处理下显著高于其他处理, 而在其他处理间均无显 著变化(图1f); 叶生物量比随着光照的降低显著增 加, 且在低光高水处理下显著高于高光低水处理 (图1 $\mathrm{g}$ )。本地种的根生物量比在高光高水处理下显 著大于低光高水处理, 在其他各处理间无显著变化 $(P<0.05$, 图1e); 茎生物量比在低光高水处理下显 著高于高光低水处理, 其他各处理间变化不显著 (图1f); 叶生物量比在高光低水处理下达最大值, 且显著高于高光高水与低光高水处理, 其他各处理 间无显著差异(图1g)。入侵种在高光下投入到地下 吸收器官根的资源较多, 而在低光下投入到光能捕 获器官叶的资源较多, 以尽可能多地利用有限资源, 表现出较好的分配调节策略。

\section{3 光照和水分变化对入侵种与本地种光合生理 参数的影响}

多因素方差分析结果表明, 仅光照对光合参数 的影响显著(表1), 说明光合参数在不同光照处理间 有差异, 在不同水分处理间差异不显著。光照与来 源交互和水分与来源交互对光合参数的影响均不 显著, 说明入侵种与本地种的光合生理特性对光照 和水分变化的响应一致。入侵种与本地种的光合参 数均随着光照的降低显著降低, 且多数处理下光合 参数在入侵种与本地种间无显著差异(图2)。

\section{$2.4 入$ 侵种与本地种对光照和水分响应的表型可 塑性}

不同光照和水分处理下入侵种与本地种的表 型可塑性指数结果表明, 入侵种与本地种对水分响 应的可塑性指数较小, 对光照响应的可塑性指数较 高, 且入侵种多数变量对光照响应的可塑性指数高 于本地种, 各变量的平均可塑性指数也高于本地种 (表2)。这说明相对于水分, 光照对入侵种与本地种 的影响作用更大, 同时入侵种对光照响应的表型可 塑性高于本地种。

\section{表1 光照、水分、物种来源及其交互作用对鬼针草属入侵种和本地种形态、生长及光合参数的影响( $F$ 值)}

Table 1 Effects of light, water, origin and their interactions on morphology, growth and photosynthetic parameters of invasive and native Bidens species ( $F$-values)

\begin{tabular}{lllllllll} 
变量 Variables & $\begin{array}{l}\text { 光照 Light } \\
(\mathrm{L})(d f=1)\end{array}$ & $\begin{array}{l}\text { 水分 Water } \\
(\mathrm{W})(d f=1)\end{array}$ & $\begin{array}{l}\text { 来源 Origin } \\
(\mathrm{O})(d f=1)\end{array}$ & $\begin{array}{l}\text { 物种(来源) Species } \\
(\text { origin })(d f=2)\end{array}$ & $\begin{array}{l}\mathrm{L} \times \mathrm{W} \\
(d f=1)\end{array}$ & $\begin{array}{l}\mathrm{L} \times \mathrm{O} \\
(d f=1)\end{array}$ & $\begin{array}{l}\mathrm{W} \times \mathrm{O} \\
(d f=1)\end{array}$ & $\begin{array}{l}\mathrm{L} \times \mathrm{W} \times \mathrm{O} \\
(d f=1)\end{array}$ \\
\hline 株高 Plant height & $194.98^{* * *}$ & $39.31^{* * *}$ & 0.39 & $30.70^{* * *}$ & $50.92^{* * *}$ & $8.11^{* *}$ & 1.24 & $5.96^{*}$ \\
比叶面积 $S L A$ & $467.90^{* * *}$ & $11.36^{* *}$ & 15.32 & 0.78 & $6.36^{*}$ & $7.38^{*}$ & 0.08 & 0.08 \\
总生物量 Total biomass & $494.65^{* * *}$ & $76.66^{* * *}$ & 0.544 & $25.33^{* * *}$ & $73.15^{* * *}$ & $11.2^{* *}$ & 3.45 & 3.7 \\
相对生长速率 $R G R$ & $1,747.95^{* * *}$ & $29.13^{* * *}$ & 0.89 & $101.44^{* * *}$ & $33.78^{* * *}$ & $5.70^{*}$ & 3.37 & 1.02 \\
根生物量比 $R M R$ & $46.92^{* * *}$ & 0.03 & 0.09 & $9.07^{* * *}$ & 3.16 & $15.43^{* * *}$ & 0.04 & 0.001 \\
茎生物量比 $S M R$ & 0.101 & $9.09^{* *}$ & 4.27 & 2.44 & 1.30 & $11.05^{* *}$ & 0.06 & 0.03 \\
叶生物量比 $L M R$ & $5.53^{*}$ & $8.07^{* *}$ & 5.13 & 1.49 & 3.19 & $22.38^{* * *}$ & 0.02 & 0.03 \\
光补偿点 $L C P$ & $220.98^{* * *}$ & 0.01 & 0.01 & 2.17 & 0.91 & 0.57 & 0.10 & 0.005 \\
光饱和点 $L S P$ & $117.78^{* * *}$ & 0.06 & 0.57 & 0.61 & 0.05 & 0.60 & 0.73 & 0.03 \\
最大净光合速率 $A_{\max }$ & $132.73^{* * *}$ & 0.08 & 0.16 & 0.81 & 0.60 & 0.64 & 0.44 & 0.10 \\
暗呼吸速率 $R_{\text {day }}$ & $119.90^{* * *}$ & 0.064 & 0.036 & $3.45^{*}$ & 0.002 & 0.054 & 0.052 & 0.279 \\
\hline SLA, Spcifi
\end{tabular}

$S L A$, Specific leaf area; $R G R$, Relative growth rate; $R M R$, Root mass ratio; SMR, Stem mass ratio; $L M R$, Leaf mass ratio; $L C P$, Light compensation point; $L S P$, Light saturation point; $A_{\max }$, Maximum net photosynthetic rate; $R_{\text {day }}$, Dark respiration rate. $* P<0.05, * * P<0.01, * * * P<0.001$. 

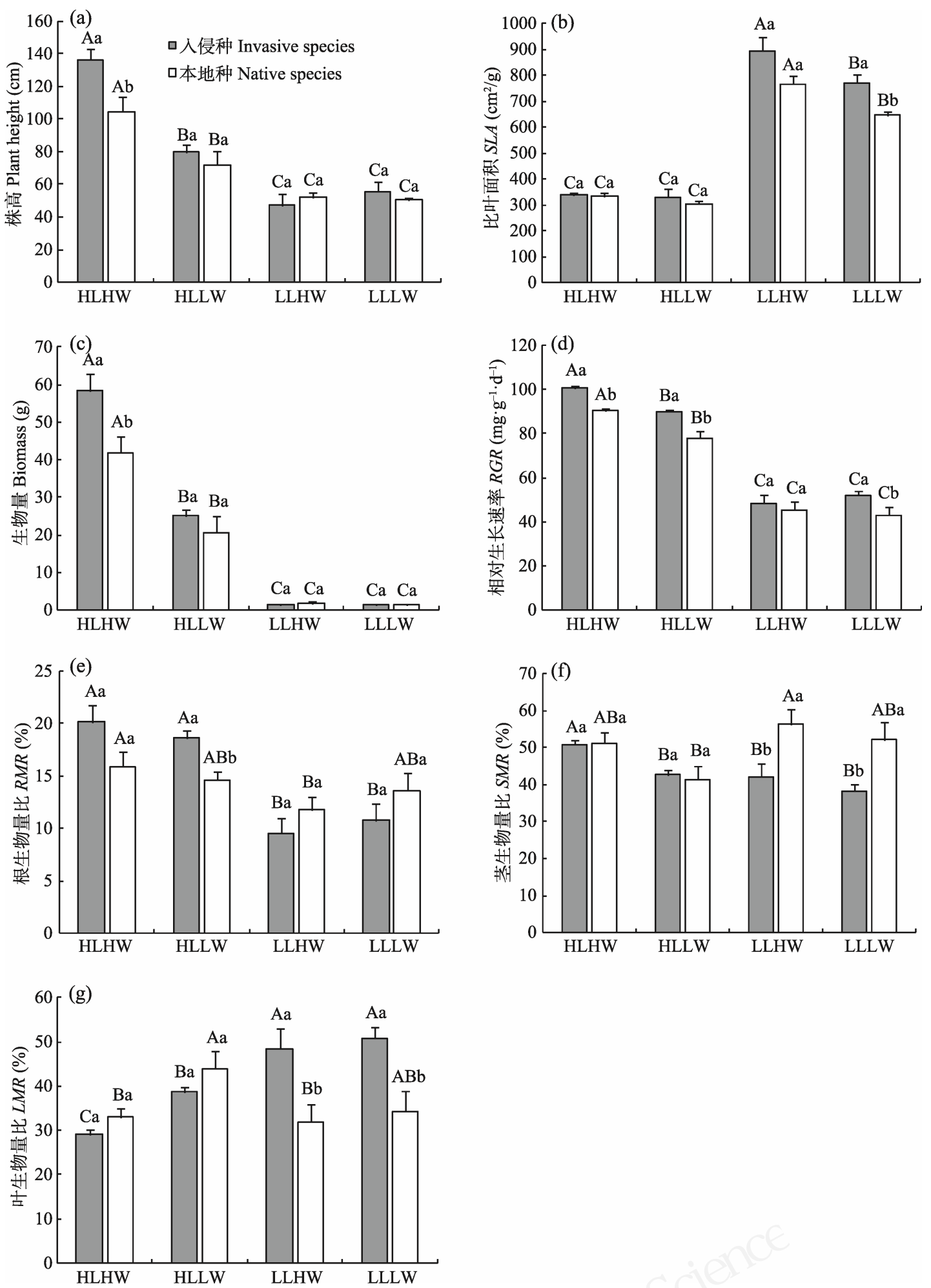

图1 不同光照和水分条件下鬼针草属入侵种与本地种的形态、生长及生物量分配特征(平均值 \pm 标准误)。不同大、小写字母 分别代表物种在不同处理下和相同处理下入侵种和本地种间差异显著 $(P<0.05)$ 。 HLHW: 高光高水; HLLW: 高光低水; LLHW: 低光高水; LLLW: 低光低水。

Fig. 1 Morphology, growth and biomass allocation characteristics of the invasive and native Bidens species under different light and water conditions (means $\pm S E$ ). Different capital and small letters indicate significant differences among treatments for the same species and differences between invasive and native species at the same treatment, respectively $(P<0.05)$. HLHW, High light and high water; HLLW, High light and low water; LLHW, Low light and high water; LLLW, Low light and low water; SLA, Specific leaf area; $R G R$, Relative growth rate; $R M R$, Root mass ratio; SMR, Stem mass ratio; LMR, Leaf mass ratio. 

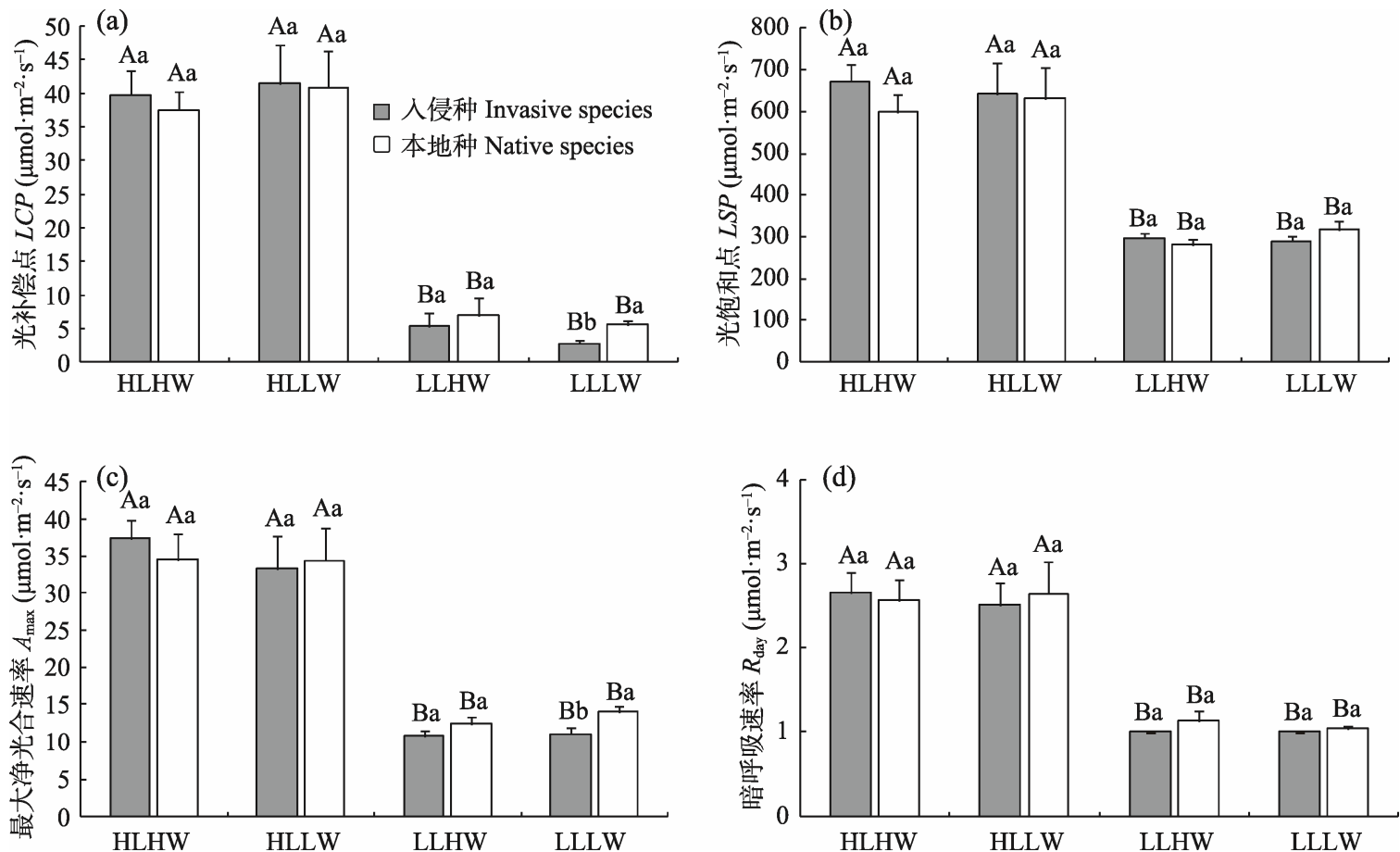

图2 不同光照和水分条件下鬼针草属入侵种和本地种的光合参数(平均值土标准误)。不同大、小写字母分别代表物种在不同 处理下和相同处理下入侵种和本地种间差异显著 $(P<0.05)$ 。HLHW: 高光高水; HLLW: 高光低水; LLHW: 低光高水; LLLW：低光低水。

Fig. 2 Photosynthetic parameters of the invasive and native Bidens species under different light and water conditions (means $\pm S E$ ). Different capital and small letters indicate significant differences among treatments for the same species and differences between invasive and native species at the same treatment, respectively $(P<0.05)$. HLHW, High light and high water; HLLW, High light and low water; LLHW, Low light and high water; LLLW, Low light and low water; LCP, Light compensation point; LSP, Light saturation point; $A_{\max }$, Maximum net photosynthetic rate; $R_{\text {day }}$, Dark respiration rate.

表2 鬼针草属入侵种和本地种各变量对光照和水分响应的表型可塑性指数

Table 2 Phenotypic plasticity indices for the variables of invasive and native Bidens species to light and/or water conditions

\begin{tabular}{|c|c|c|c|c|c|c|c|c|}
\hline \multirow[t]{3}{*}{ 变量 Variables } & \multicolumn{4}{|c|}{ 入侵种 Invasive species } & \multicolumn{4}{|c|}{ 本地种 Native species } \\
\hline & \multicolumn{2}{|l|}{ 光照 Light } & \multicolumn{2}{|c|}{ 水分 Water } & \multicolumn{2}{|l|}{ 光照 Light } & \multicolumn{2}{|l|}{ 水分 Water } \\
\hline & $\begin{array}{l}\text { 高水处理 } \\
\text { High water }\end{array}$ & $\begin{array}{l}\text { 低水处理 } \\
\text { Low water }\end{array}$ & $\begin{array}{l}\text { 高光处理 } \\
\text { High light }\end{array}$ & $\begin{array}{l}\text { 低光处理 } \\
\text { Low light }\end{array}$ & $\begin{array}{l}\text { 高水处理 } \\
\text { High water }\end{array}$ & $\begin{array}{l}\text { 低水处理 } \\
\text { Low water }\end{array}$ & $\begin{array}{l}\text { 高光处理 } \\
\text { High light }\end{array}$ & $\begin{array}{l}\text { 低光处理 } \\
\text { Low light }\end{array}$ \\
\hline 株高 Plant height & 0.65 & 0.302 & 0.41 & 0.15 & 0.5 & 0.298 & 0.31 & 0.03 \\
\hline 比叶面积 SLA & 0.62 & 0.57 & 0.02 & 0.135 & 0.57 & 0.53 & 0.09 & 0.16 \\
\hline 总生物量 Total biomass & 0.98 & 0.94 & 0.57 & 0.09 & 0.96 & 0.93 & 0.51 & 0.24 \\
\hline 根生物量比 $R M R$ & 0.53 & 0.42 & 0.08 & 0.12 & 0.26 & 0.07 & 0.08 & 0.14 \\
\hline 茎生物量比 SMR & 0.17 & 0.1 & 0.16 & 0.09 & 0.1 & 0.2 & 0.19 & 0.07 \\
\hline 叶生物量比 $L M R$ & 0.4 & 0.24 & 0.25 & 0.05 & 0.03 & 0.22 & 0.25 & 0.07 \\
\hline 相对生长速率 $R G R$ & 0.52 & 0.42 & 0.1 & 0.07 & 0.5 & 0.45 & 0.14 & 0.05 \\
\hline 最大净光合速率 $A_{\max }$ & 0.71 & 0.67 & 0.11 & 0.03 & 0.64 & 0.59 & 0.005 & 0.12 \\
\hline 光补偿点 $L C P$ & 0.87 & 0.93 & 0.04 & 0.48 & 0.81 & 0.86 & 0.08 & 0.21 \\
\hline 光饱和点 $L S P$ & 0.56 & 0.55 & 0.05 & 0.03 & 0.53 & 0.5 & 0.05 & 0.11 \\
\hline 平均值 Mean value & 0.6 & 0.51 & 0.18 & 0.12 & 0.49 & 0.46 & 0.17 & 0.12 \\
\hline
\end{tabular}

$S L A$, Specific leaf area; $R M R$, Root mass ratio; $S M R$, Stem mass ratio; $L M R$, Leaf mass ratio; $R G R$, Relative growth rate; $A_{\max }$, Maximum net photosynthetic rate; LCP, Light compensation point; LSP, Light saturation point. 


\section{3 讨论}

\section{1 形态、生长及生理性状与鬼针草属入侵种的入 侵性}

外来物种的入侵性在很大程度上由其自身的 生物学特性决定, 研究与生长相关的性状值有助于 解释其入侵性(Py̌sek \& Richardson, 2007; Godoy et $\mathrm{al}, 2011)$ 。在有利的条件下, 入侵种往往要比本地种 积累的生物量更多 (Graeme et al, 2008; Zheng et al, 2009)。如果外来物种能在某一类型的生境中定殖并 产生较高的生物量, 说明该物种在此生境中的入侵 性较高(王坤等, 2009)。Zheng等(2009)认为较大的生 物量和较快的相对生长速率是紫茎泽兰具有入侵 性的重要原因。本研究中, 在高光高水条件下, 鬼 针草属入侵种的生物量和相对生长速率均显著高 于本地种, 这可能是其入侵性之一。此外, 在有利 的光照和水分条件下, 鬼针草属入侵种与本地种的 比叶面积没有差异, 但在低光低水条件下, 入侵种 的比叶面积显著高于同属本地种。Feng和Fu (2008) 认为较大的比叶面积能降低叶的构建成本, 提高对 资源的捕获和利用效率。低光下较大的比叶面积可 能提高了鬼针草属入侵种对光资源的捕获能力和 对不利环境的适应性。

较大的最大净光合速率可能是外来植物成功 入侵的重要特性(Feng, 2008)。Zheng等(2009)发现入 侵种紫茎泽兰在所有光照条件下的最大净光合速 率显著高于同属本地种异叶泽兰(E. heterophyllum) 和白头婆(E. japonicum)。但我们的研究并没发现鬼 针草属入侵种的最大净光合速率高于同属本地种, 而且其他光合参数在入侵种与本地种之间也没有 显著差异。Hou等(2015)对旋花科和菊科入侵种和非 入侵种的研究也发现, 入侵种和非入侵种的最大净 光合速率没有显著差异。入侵种铁扫帚(Lespedeza cuneata) 与其同属本地种胡枝子(L. capitata)也表现 出相似的生理生态特性(Smith \& Knapp, 2001)。这 表明相对于光合生理特征, 形态与生长特征可能对 鬼针草属入侵种的入侵性更重要。

\section{2 生物量分配策略与鬼针草属入侵种的入侵性}

植物的生物量分配策略可能会直接影响植物 获取资源及对环境的适应能力(Johnson et al, 2008; 间帮国等, 2016)。与本地种相比, 入侵种在光照增
加时分配相对较多的生物量给地下部分或者在光 照受限时分配较多的生物量给地上部分, 以尽可能 地保证它们在任何不利环境条件下都可以成功地 捕获资源 (Ruprecht et al, 2014)。本研究中, 入侵种 在高光处理下分配更多的生物量给地下根, 增加对 地下部分的投入, 促进了对水分和养分的吸收; 在 低光处理下分配更多的生物量给地上光合结构叶, 增加对光资源的捕获, 保证其在不利条件下利用较 多的光资源。总体上, 本地种各构件的生物量分配 在不同光照和水分处理下变化不显著, 表现出入侵 种与本地种的生物量分配特征对异质环境响应的 差异性。相对于本地种, 入侵种在不同环境条件下 通过灵活调整各功能构件的生物量分配以尽可能 多地捕获和利用现有资源的生物量分配策略可能 也是其资源竞争的优势特征, 这可能是其较快适应 入侵地生境, 从而成功定居的原因。

\section{3 表型可塑性与鬼针草属入侵种的入侵性}

光照是植物生长最重要的资源, 它影响着植物 的营养生长和繁殖生长(Poorter, 2001)。本研究发现 光照对鬼针草属入侵种和本地种均有显著影响。入 侵种与本地种的形态和生长参数对光照变化的响 应有差异。相对于本地种, 入侵种各变量对光照响 应的变化幅度较大, 可塑性指数较高(表2), 表现出 较高的表型可塑性。表型可塑性被认为与外来植物 的入侵性相关(Daehler, 2003; 耿宇鹏等, 2004; Davidson et al, 2011)。具有较高表型可塑性的物种能适 应更宽广的环境条件, 能在不利条件下优化利用资 源(Annapurna \& Singh, 2003; Gupta \& Narayan, 2012)。鬼针草属入侵种多数变量对光照响应的较高 的表型可塑性既增加了它们对低光的忍耐力(较大 的比叶面积), 又使它们能积极响应高光资源(较大 的株高、生物量和相对生长速率), 提高了对资源的 利用效率, 促进其入侵。本研究中, 入侵种与本地 种对水分的响应无显著差异, 说明它们对水分的适 应性相似。野外调查也发现, 鬼针草属的这几种植 物在旱地和湿地等具有不同土壤湿度的生境中均 有分布。这说明相对于水分, 光照可能对这两种入 侵种的入侵性影响更大。

总之, 我们的研究结果表明, 较大的生长性状 值如株高、生物量和相对生长速率及表型可塑性等 可能与鬼针草属入侵种的入侵性相关。灵活的资源 分配策略使其在有利条件下表现出生长最大化, 在 
不利条件下提高对资源的捕获能力, 维持生长。这 些特性体现出入侵种对资源捕获和利用的优势。 鉴于鬼针草属入侵植物较强的环境适应能力及其 在我国急剧扩散的趋势, 应深入开展其成功入侵因 素和防控方法的研究，降低其对本地物种多样性的 威胁。

\section{参考文献}

Albert KR, Ro-Poulsen H, Mikkelsen TN, Michelsen A, van der Linden L, Beier C (2011) Interactive effects of elevated $\mathrm{CO}_{2}$, warming, and drought on photosynthesis of Deschampsia flexuosia in a temperate health ecosystem. Journal of Experimental Botany, 62, 4253-4266.

Annapurna C, Singh JS (2003) Variation of Parthenium hysterophorus in response to soil quality: implications for invasiveness. Weed Research, 43, 190-198.

Burns JH (2004) A comparison of invasive and noninvasive dayflowers (Commelinaceae) across experimental nutrient and water gradients. Diversity and Distributions. 10, 387397.

Cannell MGR, Thornley JHM (1998) Temperature and $\mathrm{CO}_{2}$ responses of leaf and canopy photosynthesis: a clarification using the non-rectangular hyperbola model of photosynthesis. Annals of Botany, 82, 883-892.

Daehler CC (2003) Performance comparisons of co-occurring native and alien invasive plants: implications for conservation and restoration. Annual Review of Ecology, Evolution, \& Systematics, 34, 183-211.

Davidson AM, Jennions M, Nicotra AB (2011) Do invasive species show higher phenotypic plasticity than native species and, if so, is it adaptive? A meta-analysis. Ecology Letters, 14, 419-431.

Deng X, Ye WH, Feng HL, Yang QH, Cao HL, Xu KY, Zhang $Y$ (2004) Gas exchange characteristics of the invasive species Mikania micrantha and its indigenous congener $M$. cordata (Asteraceae) in South China. Botanical Bulletin of Academia Sinica, 45, 213-220.

Droste T, Flory SL, Clay K (2010) Variation for phenotypic plasticity among populations of an invasive exotic grass. Plant Ecology, 207, 297-306.

Endo AT, Okuda T, Tamura M, Yasuoka Y (2001) Estimation of net photosynthetic rate based on in-situ hyperspectral data. Proceeding of Spie, 15, 214-221.

Feng YL (2008) Photosynthesis, nitrogen allocation and specific leaf area in invasive Eupatorium adenophorum and native Eupatorium japonicum grown at different irradiances. Physiologia Plantarum, 133, 318-326.

Feng YL, Fu GL (2008) Nitrogen allocation, partitioning and use efficiency in three invasive plant species in comparison with their native congeners. Biological Invasions, 10, 891-902.
Geng YP, Pan XY, Xu CY, Zhang WJ, Li B, Chen JK (2006) Phenotypic plasticity of invasive Alternathera philoxeroides in relation to different water availability, compared to its native congener. Acta Oecologica, 30, 380-385.

Geng YP, Zhang WJ, Li B, Chen JK (2004) Phenotypic plasticity and invasiveness of alien plants. Biodiversity Science, 12, 447-455. (in Chinese with English abstract) [耿宇鹏, 张文驹, 李博, 陈家宽 (2004) 表型可塑性与外来植物的 入侵能力. 生物多样性, 12, 447-455.]

Godoy O, Valladares F, Castro-Diez P (2011) Multispecies comparison reveals that invasive and native plants differ in their traits but not in their plasticity. Functional Ecology, 25, 1248-1259.

Graeme TH, Andrew JD, Gabrielle VS (2008) Predicting invasiveness in exotic species: do subtropical native and invasive exotic aquatic plants differ in their growth response to macronutrients? Diversity and Distributions, 14, 243-251.

Grotkopp E, Rejmánek M (2007) High seedling relative growth rate and specific leaf area are traits of invasive species: phylogenetically independent contrasts of woody angiosperms. American Journal of Botany, 94, 526-532.

Guo AY, Yang Q, Zhang FJ, Meng XD (2010) Effects of the leaf extract from railway beggar ticks and Ageratum conyzoides on seeds germination of upland rice. Journal of Hebei Normal University of Science \& Technololy, 24(3), 28-31. (in Chinese with English abstract) [郭艾英, 杨晴, 张风娟, 孟宪东 (2010) 三叶鬼针草和胜红蓟的叶浸提液对旱稻 种子萌发的影响. 河北科技师范学院学报, 24(3), 28-31.]

Gupta S, Narayan R (2012) Phenotypic plasticity of Chenopodium murale across contrasting habitat conditions in peri-urban areas in Indian dry tropics, is it indicative of its invasiveness? Plant Ecology, 213, 493-503.

Hao JH, Liu QQ, Qiang S (2009) Reproductive traits associated with invasiveness in Bidens Pilosa (Asteraceae). Chinese Bulletin of Botany, 44, 656-665. (in Chinese with English abstract) [郝建华, 刘倩倩, 强胜 (2009) 菊科入侵植物三 叶鬼针草的繁殖特征及其与入侵性的关系. 植物学报, 44, 656-665.]

Hong L, Shen H, Yang QH, Cao HL, Ye WH (2004) Studies on seed germination and storage of the invasive alien species Bidens pilosa L. Journal of Wuhan Botanical Research, 22, 433-437. (in Chinese with English abstract) [洪岗, 沈浩, 杨期和, 曹洪麟, 叶万辉 (2004) 外来入侵植物三叶鬼针 草种子萌发与拒藏特性研究. 武汉植物学研究, 22 , 433-437.]

Hou YP, Peng SL, Lin ZG, Huang QQ, Ni GY, Zhao N (2015) Fast-growing and poorly shade-tolerant invasive species may exhibit higher physiological but not morphological plasticity compared with non-invasive species. Biological Invasions, 17, 1555-1567.

Huang H, Guo SL (2005) Study on reproductive biology of the invasive plant Solidago canadensis. Acta Ecologica Sinica, 25, 2795-2803. (in Chinese with English abstract) [黄华, 
郭水良 (2005) 外来入侵植物加拿大一枝黄花繁殖生物 学研究. 生态学报, 25, 2795-2803.]

Johnson NC, Rowland DL, Corkidi L, Allen EB (2008) Plant winners and losers during grassland $\mathrm{N}$-eutrophication differ in biomass allocation and mycorrhizas. Ecology, 89, 2868-2878.

Mao DJ, Xie JF, Quan GM, Zhang JE (2010) Effects of Bidens pilosa aqueous extracts on germination and seedling growth of two pastures. Journal of Foshan University (Natural and Science Edition), 28(5), 7-11. (in Chinese with English abstract) [毛丹鹃, 谢俊芳, 全国明, 章家恩 (2010) 三叶鬼 针草水浸提液对两种牧草种子萌发与幼苗生长的影响. 佛山科学技术学院学报(自然科学版), 28(5), 7-11.]

McDowell SCL (2002) Photosynthetic characteristics of invasive and noninvasive species of Rubus (Rosaceae). American Journal of Botany, 89, 1431-1438.

Mozdzer TJ, Megonigal JP (2012) Jack-and-master trait responses to elevated $\mathrm{CO}_{2}$ and $\mathrm{N}$ : a comparison of native and introduced Phragmites australis. PLoS ONE, 7, e42794.

Pan YM, Liu MC, Tang SC, Wei CQ, Pu GZ, Cen YX (2012a) Effect of light intensity on the growth characteristics of Bidens pilosa. Guihaia, 32, 77-82. (in Chinese with English abstract) [潘玉梅, 刘明超, 唐赛春, 韦春强, 蒲高忠, 岑 艳喜 (2012a) 光照对三叶鬼针草生长特征的影响. 广西 植物, 32, 77-82.]

Pan YM, Tang SC, Wei CQ, Liu MC (2012b) Response of growth characteristics of Bidens pilosa L. to soil nitrogen level. Weed Science, 30, 11-16. (in Chinese with English abstract) [潘玉梅, 唐赛春, 韦春强, 刘明超 (2012b) 三 叶鬼针草生长特征对土壤氮素水平的响应. 杂草科学, 30, 11-16.]

Pan YM, Tang SC, Wei CQ, Li XQ (2016) Effects of global risks - nitrogen additions on growth and competitive relations among invasive and native congeneric species - Bidens frondosa. Polish Journal of Ecology, 64, 443-452.

Poorter L (1999) Growth response of 15 rain forest tree species to a light gradient: the relative importance of morphological and physiological traits. Functional Ecology, 13, 396-410.

Poorter L (2001) Light-dependent changes in biomass allocation and their importance for growth of rain forest tree species. Functional Ecology, 15, 113-123.

Pyšek P, Richardson DM (2007) Ecological studies: traits associated with invasiveness in alien plants, where do we stand? In: Biological Invasions (ed. Nentwig W), pp. 97-126. Springer-Verlag, Berlin \& Heidelberg.

Quan GM, Mao DJ, Zhang JE, Xie JF, Xu HQ (2011) Reproductive capacity and seed germination characteristics of Chromolaena odorata. Ecology and Environmental Sciences, 20(1), 72-78. (in Chinese with English abstract) [全 国明, 毛丹鹃, 章家恩, 谢俊芳, 徐华勤 (2011) 飞机草 的繁殖能力与种子的萌发特性. 生态环境学报, 20(1), 72-78.]

Quero JL, Villar R, Mrańön T, Zamora R (2006) Interactions of drought and shade effects on seedlings of four Quercus species: physiological and structural leaf responses. New Phytologist, 170, 819-834.

Ruprecht E, Fenesi A, Nijs I (2014) Are plasticity in functional traits and constancy in performance traits linked with invasiveness? An experimental test comparing invasive and naturalized plant species. Biological Invasions, 16, 1359-1372.

Smith MD, Knapp AK (2001) Physiological and morphological traits of exotic, invasive exotic, and native plant species in tallgrass prairie. International Journal of Plant Science, 162, 785-792.

Song LY, Peng CL, Peng SL (2009) Comparison of leaf construction costs between three invasive species and three native species in South China. Biodiversity Science, 17, 378-384. (in Chinese with English abstract) [宋莉英, 彭长 连, 彭少麟 (2009) 华南地区3 种入侵植物与本地植物叶 片建成成本的比较. 生物多样性, 17, 378-384.]

Valladares F, Wright SJ, Lasso E, Kitajima K, Pearcy RW (2000) Plastic phenotypic response to light of 16 congeneric shrubs from a Panamanian rainforest. Ecology, 81, 1925-1936.

Wang K, Yang J, Chen JK (2009) The applications of congeneric comparisons in plant invasion ecology. Biodiversity Science, 17, 353-361. (in Chinese with English abstract) [王 坤, 杨继, 陈家宽 (2009) 近缘种比较研究在植物入侵生 态学中的应用. 生物多样性, 17, 353-361.]

Wang K, Yang J, Chen JK (2010) Comparison of morphological traits between alligator weed and two congeners under different water and nutrient conditions. Biodiversity Science, 18, 615-621. (in Chinese with English abstract) [王坤, 杨继, 陈家宽 (2010) 不同土壤水分和养分条件下喜旱 莲子草与同属种生长状况的比较研究. 生物多样性, 18 , 615-621.]

Wang XH, Ji MS (2013) Photosynthetic characteristics of an invasive plant Conyza canadensis and its associated plants. Chinese Journal of Applied Ecology, 24, 71-77. (in Chinese with English abstract) [王晓红, 纪明山 (2013) 入侵植物 小飞蓬及其伴生植物的光合特性. 应用生态学报, 24 , 71-77.]

Wei CQ, Liu MC, Tang SC, Pan YM, Pu GZ (2012) Effects of light and temperature on seed germination of Eupatorium odoratum. Guihaia, 32, 527-530. (in Chinese with English abstract) [韦春强, 刘明超, 唐赛春, 潘玉梅, 蒲高忠 (2012) 光照和温度对飞机草种子萌发的影响. 广西植物, 32, 527-530.]

Yan BG, Fan B, He GX, Shi LT, Pan ZX, Li JC, Yue XW, Liu GC (2016) Biomass allocations and their response to environmental factors for grass species in an arid-hot valley. Chinese Journal of Applied Ecology, 27, 3173-3181. (in Chinese with English abstract) [闵帮国, 柇博, 何光熊, 史 亮涛, 潘志贤, 李建查, 岳学文, 刘刚才 (2016) 干热河 谷草本植物生物量分配及其对环境因子的响应. 应用生 态学报, 27, 3173-3181.] 
Yan XH, Zeng JJ, Zhou B, Wang N, Xiang HH, Kang YY (2012) Allelopathic potential of the extracts from alien invasive plant Bidens frondosa. Journal of Yangzhou University (Agriculture and Life Science Edition), 33, 88-94. (in Chinese with English abstract) [间小红，曾建军，周兵，王宁， 项欢欢, 康媛媛 (2012) 外来入侵植物大狼耙草提取物 的化感潜力. 扬州大学学报 (农业与生命科学版), 33, 88-94.]

Yan XH, Zhou B, Yin ZF, Wang N, Zhang ZG (2016) Reproductive biological characteristics potentially contributed to invasiveness in an alien invasive plant Bidens frondosa. Plant Species Biology, 31, 107-116.

Zhao XJ, Liu WY, Zhou M, Ma WZ (2009) Comparison of growth and reproduction characters among different populations of Eupatorium adenophorum in Yunnan. Acta Botanica Boreali-Occidentalia Sinica, 29, 1252-1258. (in Chinese with English abstract) [赵相健, 刘文耀, 周蒙, 马文章 (2009) 不同地理种群紫茎泽兰生长繁殖特征的比较研
究. 西北植物学报, 29, 1252-1258.]

Zheng YL, Feng YL, Liu WX, Liao ZY (2009) Growth, biomass allocation, morphology, and photosynthesis of invasive Eupatorium adenophorum and its native congeners grown at four irradiances. Plant Ecology, 203, 263-271.

Zhou CQ, Tang SC, Pan YM, Wei CQ (2015) Effects of light and temperature on germination of heteromorphic achenes of Bidens frondosa L. Journal of Tropical and Subtropical Botany, 23, 662-668. (in Chinese with English abstract) [周 超群, 唐赛春, 潘玉梅, 韦春强 (2015) 光照和温度对入 侵植物大狼耙草异形瘦果萌发的影响. 热带亚热带植物 学报, 23, 662-668.]

Zou JW, Rogers WE, Siemann E (2009) Plasiticity of Sapium sebiferum seedling growth to light and water resources: inter- and intraspecific comparisons. Basic and Applied Ecollogy, 10, 79-88.

(责任编委: 冯玉龙 责任编辑: 黄祥忠) 